V Bhargava, ${ }^{2} \mathrm{~A}$ S Chauhan, ${ }^{3} \mathrm{~S}$ Pati, ${ }^{4} \mathrm{~V}$ S Shekhawat. 'Program Officer, Technical Support Unit of Delhi State AIDS Control Society. National AIDS Control Organization, New Delhi, India; ${ }^{2}$ Team Leader, International Clinical Epidemiological Network, New Delhi, India; ${ }^{3}$ Additional Professor, Public Health Foundation of India, Bhubaneswar, India; ${ }^{4}$ roject Associate, The Action Research Unit [TARU], Surat, India

Background A significant high proportion of men engage in sexual relationships with other men has been observed in the country, which has direct linkage with the physical and the mental health status of this population. The population is more at risk when it is associated with migrant MSMs, having the characteristics of bridge population, due to associated migrant as well as MSM health related issues. Due to their unique health care needs, the population repeatedly remains devoid of basic health care services. The purpose of the study is to highlight their overlooked health issues, barriers to care and stigma associated with migrant MSMs.

Methods A cross-sectional qualitative in-depth interviews were conducted with 62 migrated MSMs in New Delhi in the Month of November-December 2012. The snow - bowling method is used to track the hidden population. Interviews were based on pre decided themes of health Issues, service availability, barriers to care and stigma associated. Data was analysed by using thematic framework approach. New emerging issues verbatim were highlighted and the case narrative has been done.

Results The majority of the participants of the study reported that they were not aware about the health care service availability even if they are willing to get screened. Most of the participants perceived that they are suffering with the mental disorders but because of stigma associated, along with the other healthcare need, they couldn't able to avail the mental health services. Occupational hazard was also reported in the majority of the cases.

Conclusion Special attention to mental health care is required along with a comprehensive package also dealing with the physical as well as the social well being of MSMs. Special attention is required for their screening since the population being migrant is much more at risk when compared to migrant and MSM individually.

\section{P6.048 REORIENTING PUBLIC SEXUAL HEALTH SERVICE TO PRIORITY POPULATIONS, STIS AND ISSUES IN NEW SOUTH WALES, AUSTRALIA, 2006-2012}

doi:10.1136/sextrans-2013-051184.1201

${ }^{1,2}$ C Bourne, 'C Murray. 'NSW STI Programs Unit, Sydney, Australia; ${ }^{2}$ School of Public Health and Community Medicine, University of NSW, Sydney, Australia

Background Reorientation of publically funded sexual health services (PFSHS) to improve access to priority populations was a key objective of the 2006 New South Wales (NSW). STI Strategy. A network of 13 PFSHS was established across NSW in mid 1990s to support STI/HIV care and prevention without a strategic framework. Priority populations identified in the Strategy now include Aboriginal people, men who have sex with men, young people, sex workers, people with HIV/AIDS, and people who inject drugs.

Methods A multidisciplinary PFSHS working group was established within the new NSW STI Programs Unit in 2007. The group coordinated the identification of need, development and promotion of resources, support tools, online and in-perons learning packages for PFSHS staff. Recognised training providers were identified to host and assist with training coordination and evaluation.

Results Many PFSHS were unfamiliar with population health principles and their role in supporting local priority populations. Defining local roles in providing sexual health services to large numbers of young people not in other priority populations was particularly challenging. A 'road show' describing STIPU's role and population health principles training was undertaken in 2007-08.
Resources developed included priority population estimation and target calculators; a roles and responsibilities statement; a priority youth discussion paper; online priority population triage training; a state-wide standard operating procedures manual. Annual reports describing priority populations accessing PFSHS, outreach clinical service and educational activities assisted with implementation evaluation. From 2006-2011, NSW PFSHS increased proportions of clinical services to Aboriginal people, men who have sex with men, people with HIV; increased triaging; increased educational activities to local communities; and increased clinical outreach activities to priority populations.

Conclusion Fears of annual reports jeopardising funding arrangements were unfounded and proved supportive of local reorientation efforts by revealing service gaps. Appropriate reorientation to priority populations is occurring in NSW PFSHS.

\section{P6.049 PHC COORDINATORS' STI AND HIV - AIDS TECHNICAL ASSISTANCE}

doi:10.1136/sextrans-2013-051184.1202

I K Dwi Harjanti, V Fardhdiani. FHI 360, Jakarta Pusat, Indonesia

PHC Coordinators' STI and HIV - AIDS Technical Assistance Background Technical teams (TA) from Indonesia FHI 360 visits to 25 health centres in four provinces in the Indonesian capital. In order to assure and improve the quality and quantity of STI and HIV-AIDS services to be Able to provide comprehensive and sustainable service. As the follow-up of previous series of events in STI and HIV-AIDS; such as HSS, OA/OI, clinical trainings on STI and HIV-AIDS. To get the baseline data for management condom.

Methods TA team consists of HIV-AIDS coordinator at Provincial Health Officer and District Health Officer, SUM 1, and some other partners such as WHO. TAs were done by direct observation, role play, staff interview, management interview, and document checking.

Results 22 out of 25 PHCs give STI \& HCT service. 9\% of the PHCs with STI\&HCT service, had met the minimum criteria for Management Component. 64\% of PHCs have written assignment with job description for the STI Team. $36 \%$ of PHCs have written STI and HIV service flow and SOP. $86 \%$ PHCs have the latest STI\&HCT guidelines from $\mathrm{MoH}$. Most of the PHCs already do Universal Precaution, but only $36 \%$ of PHCs have PEP treatment flow.

Conclusion Encourage STI, PITC and VCT implementation at the PHCs with trained staffs. Prepare new staff to replace staff who will retire or move. Use all the latest form for recording and reporting HCT activities, and fill the form completely. Distribute guidelines to the PHCs. Re-set the counselling room and prepare counselling kit. Help the PHCs in developing internal referral flow between services. Increase the capacity of counsellor for other skill. Encourage counsellor to do condom use demonstration during counselling session.

\section{P6.050 EVALUATION OF COMPLIANCE WITH PREVENTION OF MOTHER-TO-CHILD TRANSMISSION OF HIV NATIONAL PROTOCOL IN PMTCT SITES IN KINSHASA, DEMOCRATIC REPUBLIC OF CONGO}

doi:10.1136/sextrans-2013-051184.1203

R M Amboko. University of Kwazulu natal, Durban, South Africa

Background Nearly $91 \%$ of all pregnant women living with HIV worldwide live in 25 countries, including the Democratic Republic of Congo (DRC). Even although the DRC implemented the PMTCT programme in 2001, the prevalence of HIV in pregnant women was 
$4.3 \%$ in 2009 and the prevalence of HIV in newborns was $23.3 \%$ in 2010. To date, no study has been done in the DRC, specifically Kinshasa, to explore the PMTCT national protocol.

Purpose The study aimed at evaluating compliance with the PMTCT national protocol in the selected PMTCT sites of Kinshasa Methodology A quantitative approach was employed in this study with a total of 76 health care providers, specifically nurses, and 179 records of HIV + women in 18 PMTCT sites selected in Kinshasa. A health care provider self-reporting questionnaire and review of the records of HIV + women were used for compliance assessment. Data collected was analysed using the SSPS package, Version 19.0 and MS Excel 2007.

Results This study found that nurses and HIV+ women were non-compliant (less than $80 \%$ ) with the PMTCT national protocol. The score of compliance/non-compliance decreased through the continuum of PMTCT care with a peak in labour and delivery for HIV + women. Some factors were associated with nurses and HIV+ women's compliance or non-compliance with PMTCT national protocol.

Conclusion The non-compliancy of nurses and HIV+ women found in this study goes beyond the improvement of compliance with PMTCT national protocol and necessitates full and sustainable integration of PMTCT in maternal, newborn and child health services.

\section{P6.051 COST-EFFECTIVENESS OF THE COMPLEX HIV PROGRAMME AMONG THE INJECTING DRUG USERS (IDU) IN THE CITY OF BARNAUL, ALTAI TERRITORY, RUSSIA}

doi:10.1136/sextrans-2013-051184.1204

E Demyanenko, N Vagaitseva. NGO "AntiAIDS-Siberia", Barnaul, Russian Federation

Background In 2011 the NGO "AntiAIDS-Siberia" estimated the cost-effectiveness of the complex HIV programme among the IDU in Barnaul.

Tasks:

- to assess the prevalence of HIV, HCV, related risk behaviour among the PP clients and comparison group;

- to assess and compare the cost of PP, ART and HCV treatment.

\section{Methods}

- descriptive and analytical method of epidemiological analysis of HIV sickness rate;

- bio-behavioural survey among the IDU in Barnaul, 2011;

- mathematical analysis of HIV treatment costs in compliance with the standards.

Results Different preventive components such as voluntary counselling and testing, social and medical support of the IDU, condom and injecting equipment distribution and safe skills building have been implemented in Barnaul. The average cost of HIV-prevention programme among the IDU in Barnaul is $\$ 35000$ per year. At the average 1500 IDU are covered by PP per year, so the average expenditure for PP is $\$ 23$ per 1 client. Average annual cost of ART per patient is $\$ 11356$, cost of HCV treatment (Pegasys + Ribavirin) $\$ 19000$.

According to the results of bio-behavioural survey 3 out of 150 PP clients are HIV positive (2\%), 121 (80.7\%) have HCV. In comparison group 31 out of 150 have HIV (20.7\%), 130 (86.7\%) have $\mathrm{HCV}$. If we extrapolate from the data to all IDU covered by PP in 2011, we can conclude that PP help to prevent 280 IDU from infecting HIV and HCV. It saves $\$ 3000000$ per year in the budget, which are needed to provide ART and HC treatment. At an average 1 HIV IDU infects 4 partners during a year. Prevention of 1120 cases saves $\$ 3000000$ per year in the budget which is needed to provide ART.
Conclusion Preventive programmes cost the government much lower than HIV and HCV treatment and can be considered rather effective.

\section{P6.052 INTEGRATING FAMILY PLANNING SERVICES INTO HIV COMPREHENSIVE CARE AND TREATMENT CLINICS: A CASE OF THE AIDS SUPPORT ORGANIZATION (TASO) TORORO}

doi:10.1136/sextrans-2013-051184.1205

S Okoboi, L Namwanje, R Oceng. The AIDS Support Organization, Tororo, Uganda

Issue: More than 1.4 million women in Uganda report wanting to delay pregnancy, space their children, or stop childbearing altogether but that they are not currently using any contraceptive method. In late 2010, TASO integrated family planning services into its routine comprehensive HIV care clinics with aim of making FP services accessible, prevention of unintended pregnancies and reduction of mother to child transmission.

Description The intervention consisted of continuous sensitization meetings with staff and clients to explain the new intervention and ask for support. TASO staff identified the integration of condoms, oral, emergency contraceptive pills, injectable contraceptives and inplants as a feasible beginning for on-site FP services. Clients interested in IUD and permanent methods were referred to Tororo Hospital's FP clinic. Developed plans for integration including, how to obtain commodities, and triplicate referrals system

Results Overall, the frequency of modern method use among female clients increased from $6 \%$ in Ist quarter to $28 \%$ 2nd quarter to $41 \% 3$ rd quarter and finally to $49 \%$ in 4 th quarter of 2011 . Condom use increased from 8\% to 80\%. A 2012 staff evaluation showed that the majority of providers (92\%) and clients (97\%) felt that adding FP did not affect the quality of providing care and treatment services, and that the level of integration was appropriate and costeffective, added the benefits of reducing unintended pregnancy, reducing mother-to-child transmission of HIV, improving ease in talking about sex and fertility desires with clients, strengthened staff skills. Follow-up of clients using FP services offered an additional opportunity for adherence counselling.

Next step: Incorporating family planning (FP) into HIV care and treatment services is a promising model for integrating FP and HIV services in clinics with existing strong systems to accommodate added services.

\section{P6.053 EVALUATION OF COST \& EFFICIENCY OF ALTERNATIVE SERVICE DELIVERY MODELS FOR PROVIDING STI SERVICES TO HIGH RISK INDIVIDUALS IN INDIA}

doi:10.1136/sextrans-2013-051184.1206

'D L Delhikar, ${ }^{2} \mathrm{~F}$ Homan, ${ }^{1} \mathrm{~A}$ Das. ${ }^{1} \mathrm{FH}$ 360, New Delhi, India; ${ }^{2} \mathrm{FHI} 360$, North Carolina, NC, United States

Background Avahan, a large-scale HIV prevention programme, provides STI services to over 300,000 high risk individuals (HRIs) in six states of India through six different service delivery models varying by provision site (programme operated vs. contract provider) and use of outreach activities. This study aims to determine the cost-efficiency of alternative STI service delivery models.

Methods Data from the period between January to June 2010 was collected retrospectively from a stratified random sample of 30 selected NGO/CBOs, providing STI services to HRIs. Service statistics were obtained from Avahan's Computerized Management Information System, and cost data from the NGO/CBOs financial records. Cost and outcome performance from the $\mathrm{NGO/CBOs} \mathrm{were}$ annualised and standardised to reflect a high risk group size of 1,000. Analysis was done from a service delivery perspective and did not 